\title{
A paradigm shift
}

L ast year the American Heart Association published new guidelines on the prevention of infectious endocarditis. ${ }^{1}$ They represented a radical change in the previous guidelines. After an extensive review of the evidence, the multidisciplinary group noted that even if antibiotic cover was given as prophylaxis for dental procedures in a $100 \%$ effective way, it would only prevent an extremely small number of cases of infective endocarditis. The risk of acquiring infective endocarditis from the common bacteraemia that occurs with daily activities, such as brushing the teeth, was much greater than that from bacteraemia associated with occasional dental procedures. The group therefore concluded that antibiotic prophylaxis for dental procedures was only reasonable for those patients with the highest risk of an adverse outcome from infective endocarditis. It was not recommended for patients with the highest risk of acquiring infective endocarditis. The group they defined as having the highest risk of an adverse outcome and therefore requiring cover includes patients with unrepaired cyanotic heart disease, repaired disease with prosthetic material or device in situ for the first six months after the procedure and repaired congenital heart disease where there is a residual defect adjacent to a prosthetic patch or device. It also includes patients who have previously had infective endocarditis and those with prosthetic cardiac valves.

Application of these guidelines in practice will mean that many fewer patients have antibiotic prophylaxis for dental procedures. The group recognised that these recommendations will be difficult for some patients and their carers to accept. If something has been the right thing to do for more than fifty years why has the advice suddenly changed? The answer of course is that much of what we do as part of our daily clinical practice is based on consensus rather than a critical evaluation of the evidence. The evidence to support the widespread use of antibiotic prophylaxis for dental procedures was always weak, but the consensus it was the right thing to do was strong and difficult to challenge. The American Heart Association reappraisal of the evidence is welcome, their advice is clear and many will want to follow it.

Not me however, because things are never that simple. Here in England, the National Institute for Health and Clinical Excellence has undertaken a similar authoritative review of the evidence about prevention of infective endocarditis and has come up with startlingly different conclusions. ${ }^{2}$ Interestingly their analysis of the evidence is very similar to that of the American Heart Association. Their interpretation of the implications for clinical practice is not. Their conclusion is stark, "antibiotic prophylaxis against endocarditis is not recommended for people undergoing dental procedures." While they recognise that there are high-risk groups for the disease, they do not see a case for treating them any differently from low risk groups or, for that matter, from the general population. The chairman of the guideline group describes their recommendations as a "paradigm shift from current practice." Like the authors of the American Heart Association guidelines, the group recognises some professionals and patients might find such a major change in recommendation difficult to accept.

There are therefore key differences in the recommendations of the two groups. There also many things on which they agree. Patients and healthcare staff need to be aware of the increased risk of infective endocarditis in certain groups of patients and they need to be vigilant for it. The importance of good oral hygiene is emphasised. Infections that put high-risk individuals at risk for infective endocarditis should be treated promptly to minimise the risk. Antibiotic prophylaxis is not recommended for gastrointestinal or genitourinary procedures. Interestingly both groups recommend against body piercing, although the evidence for this is not clear. The two groups do advise differently for some procedures involving the respiratory tract. The English guidelines recommend against prophylaxis while the American ones 
support it for patients with a high risk of adverse outcome from endocarditis for some respiratory tract procedures.

One key comment from both groups is that there is some evidence that for much antibiotic prophylaxis the risk of adverse events, such as anaphylaxis, may be greater than any potential benefit. So, not only is there no evidence that our historical approach does no good; it might actually have done harm. This is always going to be true, clinical interventions based upon "common sense" and "consensus" rather than a critical appraisal of the evidence are potentially dangerous. These two excellent critical reviews are welcome. I would commend the guidelines to you; they are well worth study. You will of course have to decide what to do in your own practice and you may have access to local or national guidelines of your own. I will be following the English guidelines, not because I believe them to be superior, but I recognise that we do not know all the answers and my patients deserve clear advice and a consistent approach. The effect of them will I believe be profound. Not just in the advice we give about endocarditis, but also in our regimes for follow-up of patients with minor abnormalities. If they are at low risk from endocarditis, and if they do not need advice about a prophylaxis regime, do they need follow-up at all? A paradigm shift indeed.

Edward Baker Editor-in-Chief

E-mail: ctyeditor@cambridge.org

\section{References}

1. Wilson W, Taubert KA, Gewitz M, et al. Prevention of Infective Endocarditis: Guidelines From the American Association. Circulation 2007; 116: 1736-1754.

2. Prophylaxis against infectious endocarditis. National Institute for Health and Clinical Excellence March 2008. http://www.nice.org. uk/guidance/index.jsp?action $=$ byID\&o $=11938$. 